\title{
Universiteit
}

Leiden

The Netherlands

\section{Bloch theory of entangled photon generation in non-linear photonic crystals}

Irvine, W.T.M.; Dood, M.J.A. de; Bouwmeester, D.

\section{Citation}

Irvine, W. T. M., Dood, M. J. A. de, \& Bouwmeester, D. (2005). Bloch theory of entangled photon generation in non-linear photonic crystals. Physical Review A, 72(4), 043815. doi:10.1103/PhysRevA.72.043815

Version: $\quad$ Not Applicable (or Unknown)

License: $\quad$ Leiden University Non-exclusive license

Downloaded from: https://hdl.handle.net/1887/59987

Note: To cite this publication please use the final published version (if applicable). 


\title{
Bloch theory of entangled photon generation in nonlinear photonic crystals
}

\author{
William T. M. Irvine, ${ }^{*}$ Michiel J. A. de Dood, ${ }^{\dagger}$ and Dirk Bouwmeester \\ Department of Physics, University of California, Santa Barbara, California 93106, USA
}

(Received 21 April 2005; published 20 October 2005)

\begin{abstract}
We present a quantum-mechanical description of parametric down conversion and phase matching of Bloch waves in nonlinear photonic crystals. We discuss the theory in one-dimensional Bragg structures giving a recipe for calculating the down-converted emission strength and direction. We exemplify the discussion by making explicit analytical predictions for the emission amplitude and direction from a one-dimensional structure that consists of alternating layers of $\mathrm{Al}_{0.4} \mathrm{Ga}_{0.6} \mathrm{As}$ and air. We show that the emission is suitable for the extraction of polarization-entangled photons.
\end{abstract}

DOI: 10.1103/PhysRevA.72.043815

PACS number(s): 42.65.Lm, 03.67.Mn

\section{INTRODUCTION}

Entangled photon pairs play a central role in both fundamental tests of quantum mechanics and in the implementation of quantum information theory protocols [1]. They are an appealing resource for quantum communication since they propagate easily over long distances with relatively little interaction with the environment. Furthermore they have been proposed as a resource for all-optical quantum computation [2].

A popular method to produce entangled photons is by parametric down conversion in naturally birefringent nonlinear crystals. In this process "pump" photons entering a crystal decay or "down convert" into photon pairs. The role of the nonlinearity is to mediate the interaction whereas the role of the birefringence is to ensure that the process is phasematched, i.e., that the amplitudes for the down-conversion process at different points in the crystal constructively interfere. If a particular emission geometry can be achieved, photons emerging in a specific pair of directions will be entangled in polarization [3].

In a recent paper, the authors proposed the use of nonlinear photonic crystals as a source of polarization-entangled photons [4]. The scheme harnesses the higher $\chi^{(2)}$ nonlinearity of semiconductor materials (e.g., $\chi_{\mathrm{GaAs}}^{(2)}=200 \mathrm{pm} / \mathrm{V}$ [5], cf. $\left.\chi_{\mathrm{BBO}}^{(2)}=2.2 \mathrm{pm} / \mathrm{V}[6]\right)$ to mediate the down conversion and proposes to use the photonic crystal geometry to phase match the emission. The scheme has the potential of both increasing the efficiency of the process and providing an entangledphoton source that is more amenable to integration on optical chips. The scheme is fundamentally different from schemes for quasi-phase-matching in periodically poled materials, where only the $\chi^{(2)}$ is modulated periodically.

One-dimensional photonic crystals had been considered before for classical frequency conversion in the limit that the optical wavelength is much larger than the periodicity [7-9]. Two-dimensional nonlinear photonic crystals have also been considered for classical frequency conversion [10]. Following the appearance of our proposal, Ref. [11] used semiclas-

\footnotetext{
*Email address: william@physics.ucsb.edu

${ }^{\dagger}$ Present address: Huygens Laboratory, Leiden University, P.O. Box 9504, 2300 RA Leiden, The Netherlands.
}

sical coupled mode theory to calculate colinear frequency down-conversion efficiency in one-dimensional structures using numerical calculations.

In this article we present the theory that underlies our proposal. We use the Bloch-wave formalism to discuss the quantum-mechanical down-conversion process in nonlinear photonic crystals and show how to calculate the strength and direction of the down-converted emission. The theory works for photonic crystals of all dimensions. We apply the theory to one-dimensional Bragg structures, performing calculations of the down-conversion emission analytically. By plotting the calculated emission from a structure that consists of alternating layers of $\mathrm{Al}_{0.4} \mathrm{Ga}_{0.6} \mathrm{As}$ and air, we show explicitly that entangled photon pairs can be generated in a realistic structure.

The present article is structured as follows. Sections II and III discuss Bloch waves and their quantization in linear photonic crystals following essentially the work of Caticha and Caticha [12]. In Sec. IV we derive an expression for the interaction Hamiltonian and the phase-matching function that govern the down-conversion process in nonlinear photonic crystals. In Sec. V we summarize the discussion of Bloch waves in Bragg structures by Yariv and Yeh [13] extending it to obtain expressions for the Bloch-wave Fourier components. Section VI consists of a detailed discussion of the phase-matching problem in Bragg structures, giving a recipe for establishing the emission amplitude and direction from a given structure. The discussion centers on the application of our method to an example $\mathrm{Al}_{0.4} \mathrm{Ga}_{0.6} \mathrm{As} /$ Air structure. Finally we conclude and discuss extensions of the present work in Sec. VII.

\section{WAVE PROPAGATION INSIDE A LINEAR PHOTONIC CRYSTAL}

A photonic crystal is a material with a periodic variation in the index of refraction or dielectric constant. A periodic dielectric constant $\epsilon(\mathbf{r})=\epsilon(\mathbf{r}+\Lambda)$, is seen as a periodic potential by the electric field. The eigensolutions of Maxwell's equations in a medium with periodic dielectric must therefore take the form of Bloch waves. We can thus write the following expression for the four-vector potential inside the medium: 


$$
A_{\mathbf{K}, \lambda}^{\mu}(\mathbf{r}, t)=e^{-i\left(\mathbf{K} \cdot \mathbf{r}-\omega_{\mathbf{K}, \lambda} t\right)} \sum_{\mathbf{G}} \widetilde{\alpha}_{\mathbf{K}, \lambda}^{\mu}(\mathbf{G}) e^{i \mathbf{G} \cdot \mathbf{r}} .
$$

$\mathbf{K}$ represents the Bloch momentum (and will be taken to lie within the first Brillouin zone), the index $\lambda$ runs over the two polarizations and the various branches of the dispersion relation, $\mathbf{G}$ represents a reciprocal lattice vector and $\mu$ runs from 0 to 3 . The bold face represents a Cartesian vector and only the real part of $A^{\mu}$ is of physical significance. The sum defines a periodic envelope field $\alpha_{\mathbf{K}, \lambda}^{\mu}(\mathbf{r})=\alpha_{\mathbf{K}, \lambda}^{\mu}(\mathbf{r}+\Lambda)$ with Fourier coefficients $\widetilde{\alpha}_{\mathbf{K}, \lambda}(\mathbf{G})$. There are two natural generalizations of the Coulomb gauge: $\epsilon(r) \boldsymbol{\nabla} \cdot \mathbf{A}=0$ and $\boldsymbol{\nabla} \cdot[\boldsymbol{\epsilon}(r) \mathbf{A}]$ $=0$ that reduce to the uniform dielectric Coulomb gauge $\boldsymbol{\nabla} \cdot \mathbf{A}=0$. In the gauge $\boldsymbol{\epsilon}(r) \boldsymbol{\nabla} \cdot \mathbf{A}=0$, Maxwell's equations are given by [12]

$$
\begin{gathered}
\partial_{t} A^{0}=\epsilon^{-1}(r) \nabla^{2} \mathbf{A}-\partial_{t}^{2} \mathbf{A}, \\
\nabla^{2} A^{0}=-\epsilon^{-1}(r) \boldsymbol{\nabla} \epsilon(r) \cdot\left(\partial_{t} \mathbf{A}+\nabla A^{0}\right) .
\end{gathered}
$$

Solving these equations with the ansatz (1) amounts to finding the dispersion relation between $\omega$ and $\mathbf{K}$ and an expression for $\alpha_{\mathbf{K}, \lambda}^{\mu}(\mathbf{r})$ or its Fourier coefficients $\widetilde{\alpha}_{\mathbf{K}, \lambda}^{\mu}(\mathbf{G})$. The most striking consequence of the presence of a periodic potential is the formation of frequency regions, known as "stop bands" in which no propagating solution exists. Close to these regions, the dispersion is strongly modified and $\alpha_{\mathbf{K}, \lambda}(\mathbf{r})$ takes the form of a standing wave. Away from these regions $\alpha_{\mathbf{K}, \lambda}(\mathbf{r})$ recovers its plane-wave form but the dispersion relation can differ considerably from that in a uniform dielectric in a way that can be tuned by changing the geometry and strength of the periodic modulation. The tunable dispersion relation will play a crucial role in this paper.

The electric and magnetic field can be derived from the vector potential in the usual way:

$$
\begin{gathered}
\mathbf{E}_{\mathbf{K}, \lambda}=-\boldsymbol{\nabla} \phi_{\mathbf{K}, \lambda}-\partial_{t} \mathbf{A}_{\mathbf{K}, \lambda}, \\
\mathbf{B}_{\mathbf{K}, \lambda}=\boldsymbol{\nabla} \times \mathbf{A}_{K, \lambda},
\end{gathered}
$$

where $\phi_{\mathbf{K}, \lambda}=A_{\mathbf{K}, \lambda}^{0}$ and $\mathbf{A}=\left(A_{\mathbf{K}, \lambda}^{1}, A_{\mathbf{K}, \lambda}^{2}, A_{\mathbf{K}, \lambda}^{3}\right)$, giving

$$
\begin{aligned}
\mathbf{E}_{\mathbf{K}, \lambda}(\mathbf{r}, t) & =e^{-i\left(\mathbf{K} \cdot \mathbf{r}-\omega_{\mathbf{K}, \lambda} t\right)} \sum_{\mathbf{G}} \widetilde{\varepsilon}_{\mathbf{K}, \lambda}(\mathbf{G}) e^{i \mathbf{G} \cdot \mathbf{r}}, \\
\mathbf{B}_{\mathbf{K}, \lambda}(\mathbf{r}, t) & =e^{-i\left(\mathbf{K} \cdot \mathbf{r}-\omega_{\mathbf{K}, \lambda} t\right)} \sum_{\mathbf{G}} \widetilde{\beta}_{\mathbf{K}, \lambda}(\mathbf{G}) e^{i \mathbf{G} \cdot \mathbf{r}},
\end{aligned}
$$

where the Fourier coefficients $\widetilde{\varepsilon}_{\mathbf{K}, \lambda}(\mathbf{G})$ and $\widetilde{\beta}_{\mathbf{K}, \lambda}(\mathbf{G})$ are given by

$$
\begin{gathered}
\widetilde{\varepsilon}_{\mathbf{K}, \lambda}(\mathbf{G})=-i(\mathbf{K}-\mathbf{G}) \widetilde{\alpha}_{\mathbf{K}, \lambda}^{0}(\mathbf{G})-i \omega \widetilde{\alpha}_{\mathbf{K}, \lambda}(\mathbf{G}), \\
\widetilde{\beta}_{\mathbf{K}, \lambda}(\mathbf{G})=i \mathbf{G} \times \widetilde{\alpha}_{\mathbf{K}, \lambda}(\mathbf{G}) .
\end{gathered}
$$

The symbol for the Fourier coefficients of the electric field $\widetilde{\varepsilon}$ should not be confused with the dielectric constant $\epsilon(r)$.

\section{FIELD QUANTIZATION INSIDE A LINEAR PHOTONIC CRYSTAL}

Unlike sum frequency generation, parametric downconversion does not occur classically and thus is a truly quantum-mechanical phenomenon. It is thus most natural to discuss the problem in the language of the quantized electromagnetic field. The procedure for the quantization of the electromagnetic field in a medium with nonuniform dielectric differs from that in a uniform dielectric in that the equations of motion for the potential [Eqs. (2) and (3)] involve derivatives of the dielectric function. The two natural generalizations of the Coulomb gauge $\epsilon(r) \boldsymbol{\nabla} \cdot \mathbf{A}=0$ and $\boldsymbol{\nabla} \cdot[\boldsymbol{\epsilon}(r) \mathbf{A}]=0$ lead to different quantization procedures. Here we summarize the results of Caticha and Caticha [12] who used the gauge $\epsilon(r) \boldsymbol{\nabla} \cdot \mathbf{A}=0$ to quantize the electromagnetic field in a medium with periodic dielectric. They showed that the Hamiltonian is diagonal in the Bloch-wave basis and the creation operators for the field satisfy suitably modified commutation relations. The quantized field operator is given by

$$
\hat{A}^{\mu}(\mathbf{r}, t)=\sum_{\lambda} \int \frac{d^{3} K}{(2 \pi)^{3}}\left[\hat{a}(\mathbf{K}, \lambda) A_{\mathbf{K}, \lambda}^{\mu}(\mathbf{r}, t)+\text { H.c. }\right],
$$

where $A_{\mathbf{K}, \lambda}^{\mu}(\mathbf{r}, t)$ is given by Eq. (1). The Hamiltonian can be expressed in the usual form

$$
\hat{H}=\sum_{\lambda} \int \frac{d^{3} K}{(2 \pi)^{3}} \hbar \omega_{\mathbf{K}, \lambda} \hat{a}^{\dagger}(\mathbf{K}, \lambda) \hat{a}(\mathbf{K}, \lambda)
$$

and the creation and annihilation operators $\hat{a}$ and $\hat{a}^{\dagger}$ satisfy the following commutation relations:

$$
\begin{gathered}
{\left[\hat{a}(\mathbf{K}, \lambda), \hat{a}^{\dagger}\left(\mathbf{K}^{\prime}, \lambda^{\prime}\right)\right]=(2 \pi)^{3} \delta^{(3)}\left(\mathbf{K}-\mathbf{K}^{\prime}\right) \delta_{\lambda, \lambda^{\prime}},} \\
{\left[\hat{a}(\mathbf{K}, \lambda), \hat{a}\left(\mathbf{K}^{\prime}, \lambda^{\prime}\right)\right]=0,} \\
{\left[\hat{a}^{\dagger}(\mathbf{K}, \lambda), \hat{a}^{\dagger}\left(\mathbf{K}^{\prime}, \lambda^{\prime}\right)\right]=0 .}
\end{gathered}
$$

The electric and magnetic field operators derived from Eq. (8) are given by

$$
\begin{aligned}
\hat{\mathbf{E}}(\mathbf{r}, t) & =\sum_{\lambda} \int \frac{d^{3} K}{(2 \pi)^{3}}\left[\hat{a}(\mathbf{K}, \lambda) \mathbf{E}_{\mathbf{K}, \lambda}(\mathbf{r}, t)+\text { H.c. }\right], \\
\hat{\mathbf{B}}(\mathbf{r}, t) & =\sum_{\lambda} \int \frac{d^{3} K}{(2 \pi)^{3}}\left[\hat{a}(\mathbf{K}, \lambda) \mathbf{B}_{\mathbf{K}, \lambda}(\mathbf{r}, t)+\text { H.c. }\right]
\end{aligned}
$$

with $\mathbf{E}_{\mathbf{K}, \lambda}(\mathbf{r}, t)$ and $\mathbf{B}_{\mathbf{K}, \lambda}(\mathbf{r}, t)$ given by Eqs. (4) and (5). We will now use these results to derive an expression for the quantum interaction Hamiltonian and the phase-matching function.

\section{NONLINEAR PHOTONIC CRYSTAL INTERACTION HAMILTONIAN AND PHASE MATCHING}

To derive the $\chi^{(2)}$ interaction Hamiltonian for the quantized electromagnetic field, we proceed as in the case of nonlinear optical crystals with uniform dielectric and $\chi^{(2)}$ [14]. Starting with the expression for the classical interaction Hamiltonian 


$$
\epsilon_{0} \int d V \frac{1}{2} \chi_{i j k}^{(2)}(r) E^{i}(r) E^{j}(r) E^{k}(r),
$$

(where $\chi^{(2)}$ is the second order susceptibility tensor), inserting the expression for the quantized electric Bloch field $\hat{\mathbf{E}}(r, 0)$ [Eq. (12)], making the rotating wave approximation and labeling the three interacting Bloch modes by $p, 1,2$, we obtain the following quantum interaction Hamiltonian:

$$
\begin{aligned}
\hat{H}_{\mathrm{int}}= & \sum_{\lambda_{p, 1,2}} \int d^{3} K_{p, 1,2} d V \times \epsilon_{0} \chi_{i j k}^{(2)}(r) E_{p}^{* i}(r) E_{1}^{j}(r) \\
& \times E_{2}^{k}(r) \hat{a}_{\mathrm{p}} \hat{a}_{1}^{\dagger} \hat{a}_{2}^{\dagger}+\text { H.c. },
\end{aligned}
$$

where $E_{p, 1,2}$ is short for $E_{\mathbf{K}_{p, 1,2}, \lambda_{p, 1,2}}(r, 0)$ and $\hat{a}_{p, 1,2}$ is short for $\hat{a}\left(\mathbf{K}_{p, 1,2}, \lambda_{p, 1,2}\right)$. The interaction can be seen to mediate two basic processes: one in which a $p$ photon down converts into photons 1 and 2 and (H.c.) in which photons 1 and 2 up convert to photon $p$.

To calculate the time evolution of the field under this interaction Hamiltonian, we switch to the interaction picture, and evaluate the first term in the Dyson series expansion for the time evolution of an initial state $\left|\psi_{0}\right\rangle$ :

$$
\begin{aligned}
|\psi(t)\rangle= & \left(1+\sum_{\lambda_{p, 1,2}} \int \frac{d^{3} K_{p, 1,2}}{(2 \pi)^{3}} \delta\left(\omega_{p}-\omega_{1}-\omega_{2}\right) \epsilon_{0} \int d V \chi_{i j k}^{(2)}(r)\right. \\
& \left.\times E_{p}^{* i}(r) E_{1}^{j}(r) E_{2}^{k}(r) \hat{a}_{\mathrm{p}} \hat{a}_{1}^{\dagger} \hat{a}_{2}^{\dagger}+\text { H.c. }\right)\left|\psi_{0}\right\rangle .
\end{aligned}
$$

For given states $p, 1,2$, the amplitude for parametric downconversion into modes 1 and 2 is proportional to the phasematching function $\Phi(p, 1,2)$, given by

$$
\Phi(p, 1,2)=\epsilon_{0} \int d V \chi_{i j k}^{(2)} E_{p}^{* i}(r) E_{1}^{j}(r) E_{2}^{k}(r) .
$$

It is instructive to substitute the Fourier expansion of $E_{p}(r), E_{1}(r), E_{2}(r)$ [Eq. (4)] and the Fourier expansion of $\chi_{i j k}^{(2)}(r)\left[\right.$ with Fourier coefficients denoted by $\left.\widetilde{\chi}_{i j k}^{(2)}(\mathbf{G})\right]$ into the phase-matching function [Eq. (15)] to obtain

$$
\begin{aligned}
\Phi(p, 1,2)= & \sum_{\mathbf{G}_{\chi}, \mathbf{G}_{p}, \mathbf{G}_{1}, \mathbf{G}_{2}} \epsilon_{0} \widetilde{\chi}_{i j k}^{(2)}\left(\mathbf{G}_{\chi}\right) \widetilde{\varepsilon}_{p}^{* i}\left(\mathbf{G}_{p}\right) \widetilde{\varepsilon}_{1}^{j}\left(\mathbf{G}_{1}\right) \widetilde{\varepsilon}_{2}^{k}\left(\mathbf{G}_{2}\right) \\
& \times \delta^{(3)}\left(\mathbf{K}_{p}-\mathbf{K}_{1}-\mathbf{K}_{2}+\mathbf{G}_{\chi}+\mathbf{G}_{1}+\mathbf{G}_{2}-\mathbf{G}_{p}\right)
\end{aligned}
$$

which makes the conservation of Bloch quasimomentum manifest.

Equations (15) and (16) are the main result of this section. To first order in $\chi^{(2)}$ a pump photon will down convert into a superposition of all Bloch-wave pairs that satisfy conservation of energy $\left(\omega_{1}+\omega_{2}=\omega_{p}\right)$ and of Bloch quasimomentum, with an amplitude $\Phi(p, 1,2)$ given by Eq. (15) or Eq. (16). An important difference with the phenomenon of up conversion is the fact that down conversion involves all modes that phase match, whereas up conversion is more constrained: given two photons to up convert, there is typically only one mode they can up convert to. A parallel between phase matching in nonlinear crystals with uniform dielectric and
TABLE I. Phase-matching dictionary that shows how the main ideas of phase matching in nonlinear optical crystals translate to nonlinear photonic crystals.

\begin{tabular}{cc}
\hline \hline Natural nonlinear crystals & Nonlinear photonic crystals \\
\hline Plane waves & Bloch waves \\
$\mathbf{E}_{\lambda}(\mathbf{r})=e^{i \mathbf{k} \cdot \mathbf{r}}$ & $\mathbf{E}_{\mathbf{K}, \lambda}(\mathbf{r})=e^{i \mathbf{K} \cdot \mathbf{r}} \widetilde{\varepsilon}_{\mathbf{K}, \lambda}(\mathbf{G}) e^{i \mathbf{G} \cdot \mathbf{r}}$ \\
Momentum conservation & Quasimomentum conservation \\
$\mathbf{k}_{1}+\mathbf{k}_{2}=\mathbf{k}_{\mathbf{p}}$ & $\mathbf{K}_{1}+\mathbf{K}_{2}=\mathbf{K}_{\mathrm{p}}+\mathbf{G}$ \\
Natural dispersion and & Artificial dispersion and \\
birefringence & form birefringence \\
$\mathbf{k}(\omega, \hat{\mathbf{k}})=n(\omega, \hat{\mathbf{k}}) / c$ & $\mathbf{k}(\omega, \hat{\mathbf{k}})=\mathbf{K}(\omega, \hat{\mathbf{k}})$ \\
Tensor properties $\chi^{(2)}$ & Tensor properties $\chi^{(2)}$ and \\
determine amplitude & Fourier coefficients \\
& determine amplitude \\
\hline \hline
\end{tabular}

phase matching in photonic crystals is drawn in Table I which contains a dictionary for the main concepts.

The vector equation that expresses the conservation of Bloch quasimomentum

$$
\mathbf{K}_{1}+\mathbf{K}_{2}=\mathbf{K}_{p}+\mathbf{G}_{\chi}+\mathbf{G}_{1}+\mathbf{G}_{2}-\mathbf{G}_{p}
$$

shall be referred to as the phase-matching equation. For a given $\mathbf{K}_{p}$, solving the equation corresponds to finding the intersection between the dispersion surfaces $\left|\mathbf{K}_{1}\left(\hat{k}_{1}, \omega_{1}\right)\right|$ and $\left|\mathbf{K}_{2}\left(\hat{k}_{2}, \omega_{2}\right)\right|$ for photons 1 and 2 , centered on the origin and on $\left|\mathbf{K}_{p}\left(\hat{k}_{p}, \omega_{1}, \omega_{2}\right)+\mathbf{G}\right|$, where $\hat{k}$ represents a unit vector that points in the same direction as $\mathbf{K}$. To solve the equation it is therefore necessary to compute the dispersion surfaces of photons with frequencies $\omega_{1}, \omega_{2}$, and $\omega_{p}$.

For photons that satisfy the phase-matching equation, the amplitude of the process is proportional to the overlap of the waves in the nonlinear medium. As we shall see in later sections, this is most easily calculated using Eq. (15) in cases where the light field has standing wave character and using Eq. (16) when it has the character of a propagating wave. It is therefore useful to keep both these expressions in mind when solving the phase-matching problem in a given structure.

In deriving Eq. (16), the photonic crystal (interaction) volume was taken to be infinitely large. This will of course not be the case for real photonic crystals. For finite-dimensional crystals the delta functions embodying the conservation of momentum become sinc functions:

$$
\frac{\sin \left[\left(\mathbf{K}_{p}-\mathbf{K}_{1}-\mathbf{K}_{2}+\mathbf{G}_{\chi}+\mathbf{G}_{1}+\mathbf{G}_{2}-\mathbf{G}_{p}\right)_{i} L_{i}\right]}{\left(\mathbf{K}_{p}-\mathbf{K}_{1}-\mathbf{K}_{2}+\mathbf{G}_{\chi}+\mathbf{G}_{1}+\mathbf{G}_{2}-\mathbf{G}_{p}\right)_{i} L_{i}}
$$

where $L_{i}$ is the length of the photonic crystal in the $i$ th direction. This corresponds to a certain amount of allowed momentum mismatch and, as will be seen in Sec. VI, can have significant consequences.

In one- and two-dimensional photonic crystals, there are two main mechanisms by which the dispersion surfaces are modified by the presence of the crystal: form birefringence 


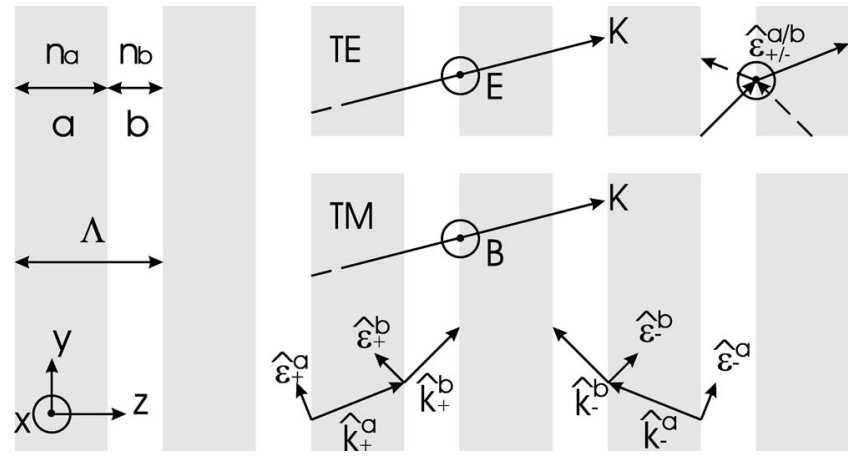

FIG. 1. Illustration of a one-dimensional photonic crystal composed of alternating layers of materials $a$ and $b$ of thickness $a$ and $b$ having refractive indices $n_{a}$ and $n_{b}$. The structure is periodic with period $\Lambda=a+b$. The axis of symmetry is taken to be the $z$ axis. There are two types of propagating polarization eigenmodes: Transverse electric (TE) and transverse magnetic (TM). TE(TM) waves have the electric(magnetic) field vector lying in a plane parallel to the interfaces between the materials.

[7] and geometric dispersion. Although the two effects are not entirely independent of each other, their origin is physically distinct. Form birefringence is the difference in the dispersion surfaces of Bloch waves that have different polarizations and arises from the different boundary conditions at the interfaces in the photonic crystal. In the long wavelength limit $(\lambda \gg \Lambda)$, this type of dispersion is the dominating one. The geometric dispersion is induced by the presence of the periodic potential and appears as the only type of dispersion for waves propagating in directions for which the boundary conditions do not break the symmetry between polarizations. In three dimensional structures the problem is more complicated since the decoupling between direction and polarization does not readily occur.

The derivations so far apply in all dimensions, however, from here on we will restrict our attention to onedimensional (Bragg) structures and discuss the analytical solution of the down-conversion phase-matching problem in detail with a view to generating entangled photon pairs. The problem in the two dimensional case is similar, however calculation of dispersion relations in two-dimensional structures has so far only been approached numerically. For a discussion of phase matching in two-dimensional photonic crystals see Ref. [10]. Reference [15] discusses the problem in a structure having a two-dimensional periodic $\chi^{(2)}$, but uniform linear dielectric.

\section{BLOCH WAVES IN (ONE DIMENSIONAL) BRAGG STRUCTURES}

In this section we summarize the results of Yariv and Yeh [13], describing the Bloch-wave solutions that propagate in one-dimensional photonic crystals (Bragg structures). We extend their results by obtaining expressions for the Blochwave Fourier coefficients and discuss the form of the Bloch waves in detail, in order to gain intuition that will be needed in the discussion of Sec. VI.

Figure 1 shows the basic structure of a one dimensional photonic crystal consisting of a series of alternating layers of materials $a$ and $b$. We adopt Cartesian coordinates with the $z$ axis aligned with the axis of symmetry. Inside each layer the field can be expressed as a superposition of forward and backward propagating plane waves, with wave vectors $\mathbf{k}$ having magnitude $|\mathbf{k}|=n \omega / c$ (where $n$ is the refractive index in the layer) and component $k_{z}^{a, b}$ along the $z$ axis. Translational symmetry in the plane perpendicular to the $z$ axis implies, through conservation of momentum, that the components of the wave vectors parallel to the planes $\left(k_{x}\right.$ and $\left.k_{y}\right)$ are equal across the boundaries, thus the $z$ components are given by

$$
k_{z}^{a, b}=\sqrt{\left(\frac{n_{a, b} \omega}{c}\right)^{2}-k_{\|}^{2}},
$$

where $k_{\|}^{2}=k_{x}^{2}+k_{y}^{2}$ and $n_{a, b}$ are the refractive indices of materials $a$ and $b$. For waves traveling at an angle to the $z$ direction, there is a natural choice for the polarization basis: transverse electric (TE) with the electric field pointing out of the plane defined by the wave vector and the $z$ axis and transverse magnetic (TM), with the electric field lying in the plane (see Fig. 1).

We can thus write down the following expressions for the electric field in the $n$th layer

$$
\begin{aligned}
\mathbf{E}_{n, \mathrm{TE}(\mathrm{M})}^{a}= & e^{i\left(\omega t-k_{y} y\right)}\left[\left(a_{n}^{+} \hat{\varepsilon}_{+}^{a}\right)_{\mathrm{TE}(\mathrm{M})} e^{-i k_{z}^{a}(z-n \Lambda)}\right. \\
& \left.+\left(a_{n}^{-} \hat{\varepsilon}_{-}^{a}\right)_{\mathrm{TE}(\mathrm{M})} e^{i k_{z}^{a}(z-n \Lambda)}\right] \\
\mathbf{E}_{n, \mathrm{TE}(\mathrm{M})}^{b}= & e^{i\left(\omega t-k_{y} y\right)}\left[\left(b_{n}^{+} \hat{\varepsilon}_{+}^{b}\right)_{\mathrm{TE}(\mathrm{M}) e^{-i k_{z}^{b}(z-n \Lambda)}}\right. \\
& \left.+\left(b_{n}^{-} \hat{\varepsilon}_{-}^{b}\right)_{\mathrm{TE}(\mathrm{M})} e^{i k_{z}^{b}(z-n \Lambda)}\right],
\end{aligned}
$$

where $\left(\hat{\varepsilon}_{ \pm}^{a, b}\right)_{\mathrm{TE}(\mathrm{M})}$ are polarization unit vectors defined in Fig. 1 and we have chosen $y$ as the off-axis direction for convenience. Matching the fields at the boundaries we obtain relations between the amplitudes in neighboring slabs, which can be further reduced to a relation between coefficients in the same material in neighboring cells:

$$
\left(\begin{array}{c}
a_{n-1}^{+} \\
a_{n-1}^{-}
\end{array}\right)=\left(\begin{array}{cc}
A & B \\
C & D
\end{array}\right)\left(\begin{array}{l}
a_{n}^{+} \\
a_{n}^{-}
\end{array}\right) .
$$

The coefficients for TE and TM waves are [13]

$$
\begin{gathered}
A_{\mathrm{TE}}=e^{i k_{z}^{a} a}\left[\cos \left(k_{z}^{b} b\right)+\frac{i}{2}\left(\frac{k_{z}^{b}}{k_{z}^{a}}+\frac{k_{z}^{a}}{k_{z}^{b}}\right) \sin \left(k_{z}^{b} b\right)\right], \\
B_{\mathrm{TE}}=e^{-i k_{z}^{a} a}\left[\frac{i}{2}\left(\frac{k_{z}^{b}}{k_{z}^{a}}-\frac{k_{z}^{a}}{k_{z}^{b}}\right) \sin \left(k_{z}^{b} b\right)\right], \\
A_{\mathrm{TM}}=e^{i k_{z}^{a} a}\left[\cos \left(k_{z}^{b} b\right)+\frac{i}{2}\left(\frac{n_{b}^{2} k_{z}^{a}}{n_{a}^{2} k_{z}^{b}}+\frac{n_{a}^{2} k_{z}^{b}}{n_{b}^{2} k_{z}^{a}}\right) \sin \left(k_{z}^{b} b\right)\right], \\
B_{\mathrm{TM}}=e^{-i k_{z}^{a} a}\left[\frac{i}{2}\left(\frac{n_{b}^{2} k_{z}^{a}}{n_{a}^{2} k_{z}^{b}}-\frac{n_{a}^{2} k_{z}^{b}}{n_{b}^{2} k_{z}^{a}}\right) \sin \left(k_{z}^{b} b\right)\right]
\end{gathered}
$$

with $C_{\mathrm{TE} / \mathrm{TM}}=B_{\mathrm{TE} / \mathrm{TM}}^{*}, D_{\mathrm{TE} / \mathrm{TM}}=A_{\mathrm{TE} / \mathrm{TM}}^{*}$. The eigenmodes of propagation can then be obtained by finding the eigenvectors 
and eigenvalues of the transfer matrix. The right-moving eigenvalue is $e^{i K_{z} \Lambda}$ with

$$
K_{z}^{\mathrm{TE} / \mathrm{TM}}\left(k_{\|}, \omega\right)=\frac{1}{\Lambda} \cos ^{-1}\left[A_{\mathrm{TE} / \mathrm{TM}}+D_{\mathrm{TE} / \mathrm{TM}}\right],
$$

where $K_{z}$ is the $z$ component of $\mathbf{K}=\left(K_{x}=k_{x}, K_{y}=k y, K_{z}\right)$ and the corresponding eigenvector is

$$
\begin{gathered}
a_{n}^{\mathrm{TE} / \mathrm{TM}}=e^{-i n K_{z}^{\mathrm{TE} / \mathrm{TM}}{ }_{\Lambda}} B_{\mathrm{TE} / \mathrm{TM}}, \\
b_{n}^{\mathrm{TE} / \mathrm{TM}}=e^{-i n K_{z}^{\mathrm{TE} / \mathrm{TM}} \Lambda}\left(e^{-i K_{z}^{\mathrm{TE} / \mathrm{TM}} \Lambda}-A_{\mathrm{TE} / \mathrm{TM}}\right)
\end{gathered}
$$

the $b_{n}^{ \pm}$coefficients are related to the $a_{n}^{ \pm} \mathrm{s}$ via $\left(b_{n}^{+}, b_{n}^{-}\right)$ $=\mathbf{M}\left(a_{n}^{+}, a_{n}^{-}\right)$, where $\mathbf{M}$ is given by

$$
\begin{gathered}
\mathbf{M}_{\mathrm{TE}}=\left(\begin{array}{cc}
\frac{\left(k_{z}^{a}+k_{z}^{b}\right)}{2 k_{z}^{b}} e^{i a\left(k_{z}^{a}-k_{z}^{b}\right)} & \frac{\left(k_{z}^{b}-k_{z}^{a}\right)}{2 k_{z}^{b}} e^{-i a\left(k_{z}^{a}+k_{z}^{b}\right)} \\
\frac{\left(k_{z}^{b}-k_{z}^{a}\right)}{2 k_{z}^{b}} e^{i a\left(k_{z}^{a}+k_{z}^{b}\right)} & \frac{\left(k_{z}^{b}+k_{z}^{a}\right)}{2 k_{z}^{b}} e^{-i a\left(k_{z}^{a}-k_{z}^{b}\right)}
\end{array}\right), \\
\mathbf{M}_{\mathrm{TM}}=\left(\begin{array}{ll}
\frac{\left(n_{b}^{2} k_{z}^{a}+n_{a}^{2} k_{z}^{b}\right)}{2 n_{a} n_{b} k_{z}^{b}} e^{i a\left(k_{z}^{a}-k_{z}^{b}\right)} & \frac{\left(n_{b}^{2} k_{z}^{a}-n_{a}^{2} k_{z}^{b}\right)}{2 n_{a} n_{b} k_{z}^{b}} e^{-i a\left(k_{z}^{a}+k_{z}^{b}\right)} \\
\frac{\left(n_{b}^{2} k_{z}^{a}-n_{a}^{2} k_{z}^{b}\right)}{2 n_{a} n_{b} k_{z}^{b}} e^{i a\left(k_{z}^{a}+k_{z}^{b}\right)} & \frac{\left(n_{a}^{2} k_{z}^{b}+n_{b}^{2} k_{z}^{a}\right)}{2 n_{a} n_{b} k_{z}^{b}} e^{-i a\left(k_{z}^{a}-k_{z}^{b}\right)}
\end{array}\right) .
\end{gathered}
$$

To relate these expressions to the Bloch-wave expressions in Secs. II and III we need to evaluate the Fourier transform of the expressions for the electric field [Eqs. (19) and (20)] and compare it to Eq. (4) (with $\mathbf{G}=n(2 \pi / \Lambda) \hat{\mathbf{z}}$ ). This is done in the Appendix, where we derive the following expression for $\widetilde{\varepsilon}_{\mathbf{K}, \lambda}$ :

$$
\begin{gathered}
\widetilde{\varepsilon}_{\mathbf{K}, \lambda}\left(\mathbf{G}=n \frac{2 \pi}{\Lambda} \hat{\mathbf{z}}\right) \\
=\frac{a}{\sqrt{2 \pi}} a_{0}^{+} \frac{\sin \left[\left(K_{z}-k_{z}^{a}-n \frac{2 \pi}{\Lambda}\right) \frac{a}{2}\right]}{\left(K_{z}-k_{z}^{a}-n \frac{2 \pi}{\Lambda}\right) \frac{a}{2}} e^{-i\left[K_{z}-k_{z}^{a}-n(2 \pi / \Lambda)\right] a / 2} \hat{\varepsilon}_{+}^{a} \\
+\frac{a}{\sqrt{2 \pi}} a_{0}^{-} \frac{\sin \left[\left(K_{z}+k_{z}^{a}-n \frac{2 \pi}{\Lambda}\right) \frac{a}{2}\right]}{\left(K_{z}+k_{z}^{a}-n \frac{2 \pi}{\Lambda}\right) \frac{a}{2}} e^{-i\left[K_{z}+k_{z}^{a}-n(2 \pi / \Lambda)\right] a / 2} \hat{\varepsilon}_{-}^{a} \\
+\frac{b}{\sqrt{2 \pi}} b_{0}^{+} \frac{\sin \left[\left(K_{z}-k_{z}^{b}-n \frac{2 \pi}{\Lambda}\right) \frac{b}{2}\right]}{\left(K_{z}-k_{z}^{b}-n \frac{2 \pi}{\Lambda}\right) \frac{b}{2}} e^{-i\left[K_{z}-k_{z}^{b}-n(2 \pi / \Lambda)\right](a+b / 2)} \hat{\varepsilon}_{+}^{b} \\
+\frac{b}{\sqrt{2 \pi}} b_{0}^{-} \frac{\sin \left[\left(K_{z}+k_{z}^{b}-n \frac{2 \pi}{\Lambda}\right) \frac{b}{2}\right]}{\left(K_{z}+k_{z}^{b}-n \frac{2 \pi}{\Lambda}\right) \frac{b}{2}} e^{-i\left[K_{z}+k_{z}^{b}-n(2 \pi / \Lambda)\right](a+b / 2)} \hat{\varepsilon}_{-}^{b} .
\end{gathered}
$$

The expressions for the dispersion relations $\left|K_{p, 1,2}\left(\hat{K}_{p, 1,2}, \omega_{p, 1,2}\right)\right|$, the field amplitude $\mathbf{E}_{p, 1,2}(r)$, and its Fourier coefficients $\widetilde{\varepsilon}_{\mathbf{K}_{p, 1,2}, \lambda_{p, 1,2}}(n(2 \pi / \Lambda))$ can be substituted into Eqs. (15) and (16) to give analytical expressions for the phase-matched emission from any given (one dimensional) nonlinear Bragg structure. The expressions are however not immediately intuitive. We therefore proceed to consider the results of this section in various situations to gain some intuition on how the Bloch-wave properties depend on the geometry of the crystal and on the properties of the materials.

Central to an intuitive understanding of Bloch-waves in a given structure is the band diagram for that structure. Figure 2 shows a band diagram for a structure of alternating layers of material $a$ with refractive index $n_{a}=1$ and material $b$ with refractive index $n_{b}=5$ (the values were chosen for illustrative purposes). Note that the labels $a, b$ shall also be used to indicate the thicknesses of materials $a, b$. The fill fraction $a / \Lambda$ of material $a$ is $\frac{1}{4}$. For simplicity, natural material dispersion was neglected; it will be included in the section that follows. The gray (white) areas correspond to combinations of $\omega$ and $k_{\|}$that propagate (do not propagate). A given combination of $\omega$ and $k_{\|}$will propagate if the corresponding $K_{\mathrm{TE} / \mathrm{TM}}\left(k_{\|}, \omega\right)$ is real. This will be the case if $\left|\frac{1}{2}(A+D)\right| \leqslant 1$. For TE waves $\frac{1}{2}(A+D)$ is given by

$$
\cos \left(k_{z}^{a} a+k_{z}^{b} b\right)+\left[1-\frac{1}{2}\left(\frac{k_{z}^{b}}{k_{z}^{a}}+\frac{k_{z}^{a}}{k_{z}^{b}}\right)\right] \sin \left(k_{z}^{a} a\right) \sin \left(k_{z}^{b} b\right),
$$

whereas for TM waves it is given by

$$
\cos \left(k_{z}^{a} a+k_{z}^{b} b\right)+\left[1-\frac{1}{2}\left(\frac{n_{b}^{2} k_{z}^{a}}{n_{a}^{2} k_{z}^{b}}+\frac{n_{a}^{2} k_{z}^{b}}{n_{b}^{2} k_{z}^{a}}\right)\right] \sin \left(k_{z}^{a} a\right) \sin \left(k_{z}^{b} b\right) .
$$

It can be seen that the extent to which $K_{z} \Lambda$ deviates from the simple linear relation $K_{z} \Lambda=k_{z}^{a} a+k_{z}^{b} b$ depends in a nontrivial way on the ratios of the refractive indices and on the width of the layers compared to the wavelength inside the medium. The term in the square brackets is independent of the width of the layers and is a measure of the strength of the modification of the dispersion relation (and thus the width of the bands). The trigonometric terms in turn depend on the layer widths in a simple way and have the primary function of determining the positions of the bands. It is instructive to consider the band structure for $k_{\|}=0$ of a crystal of fixed optical periodicity $l=n_{a} a+n_{b} b$ and varying optical fill fraction $f=n_{a} a / l$. Measuring the free space wavelength as a fraction of $l$ : $\lambda_{\text {free sp. }}=x \times l$ we obtain the following expression for $(A+D)$ :

$$
\cos \left(\frac{2 \pi}{x}\right)+\left[1-\frac{1}{2}\left(\frac{n_{a}}{n_{b}}+\frac{n_{b}}{n_{a}}\right)\right] \sin \left(\frac{2 \pi f}{x}\right) \sin \left(\frac{2 \pi(1-f)}{x}\right) .
$$

It can be seen that the effects of geometric dispersion disappear for $f / x=n$ or $(1-f) / x=m$ where $n$ and $m$ are integers. This corresponds to regions in which the scattered waves interfere destructively in the backward direction and the dispersion becomes the trivial one: $K \Lambda=k_{a} a+k_{b} b$. In these 


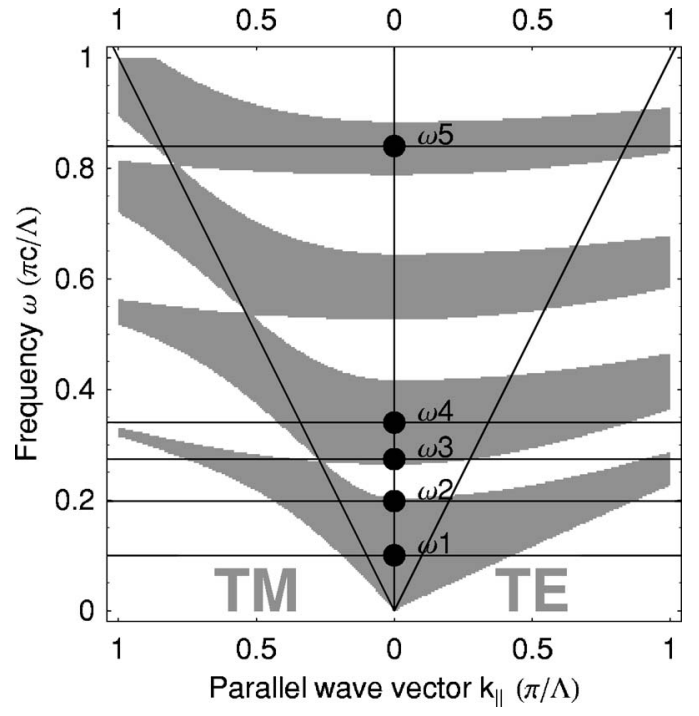

FIG. 2. Band diagram for a structure of alternating layers of material $a$ with refractive index $n_{a}=1$ and material $\mathrm{b}$ with $n_{b}=5$. The fill fraction of material $a: a / \Lambda$ is $\frac{1}{4}$. For simplicity, natural dispersion and absorption were neglected. Under this simplification, the diagram can be drawn for frequencies and parallel components of $k$ expressed as multiples of $\pi c / \Lambda$ and $\pi / \Lambda$, respectively. The points labeled $\omega_{1, \ldots, 5}$ correspond to the Bloch-wave plots of Fig. 3 and dispersion surface plots of Fig. 4.

regions, the Bloch-wave is essentially a plane wave (i.e., it has only one dominant Fourier component).

Figure 3 (left) shows the electric field amplitude and (right) the Fourier components of Bloch waves that correspond to the dots in Fig. 2. As we approach the band edges the Bloch waves can be seen to differ strongly from plane waves. At the edge of a stop band the $\omega_{2}\left(\omega_{3}\right)$ waves take the form of standing waves with the electric field concentrated in the material of higher(lower) refractive index. The magnitude of $\mathbf{K}$ is the same at these two frequencies. When estimating the down-conversion amplitude it is useful to be able to switch between configuration and Fourier space depending on the degree of localization of the Bloch waves. For waves with nonzero $k_{\|}$both the interaction strength and the position of the bands will change. In particular the difference between TE and TM waves will become increasingly marked, as can be seen by inspection of the band structure of Fig. 2. Some intuition on how the magnitude of the Bloch momentum of propagating waves varies as a function of $k_{\|}$can be gained by examining Fig. 4, where we show the dispersion surfaces for waves of frequencies $\omega_{1, \ldots, 4}$. In particular it is apparent that in the long wavelength limit, the surfaces resemble those for a uniaxial birefringent crystal. The analogy can be formalized [13] by expanding the expression for $K\left(k_{\|}, \omega\right)$ [Eq. (21)] in the limit $\lambda \gg \Lambda$ to obtain expressions for the corresponding ordinary (TE) and extraordinary (TM) refractive indices $n_{o}$ and $n_{e}$ :

$$
n_{o}^{2}=\frac{a}{\Lambda} n_{a}^{2}+\frac{b}{\lambda} n_{b}^{2}
$$
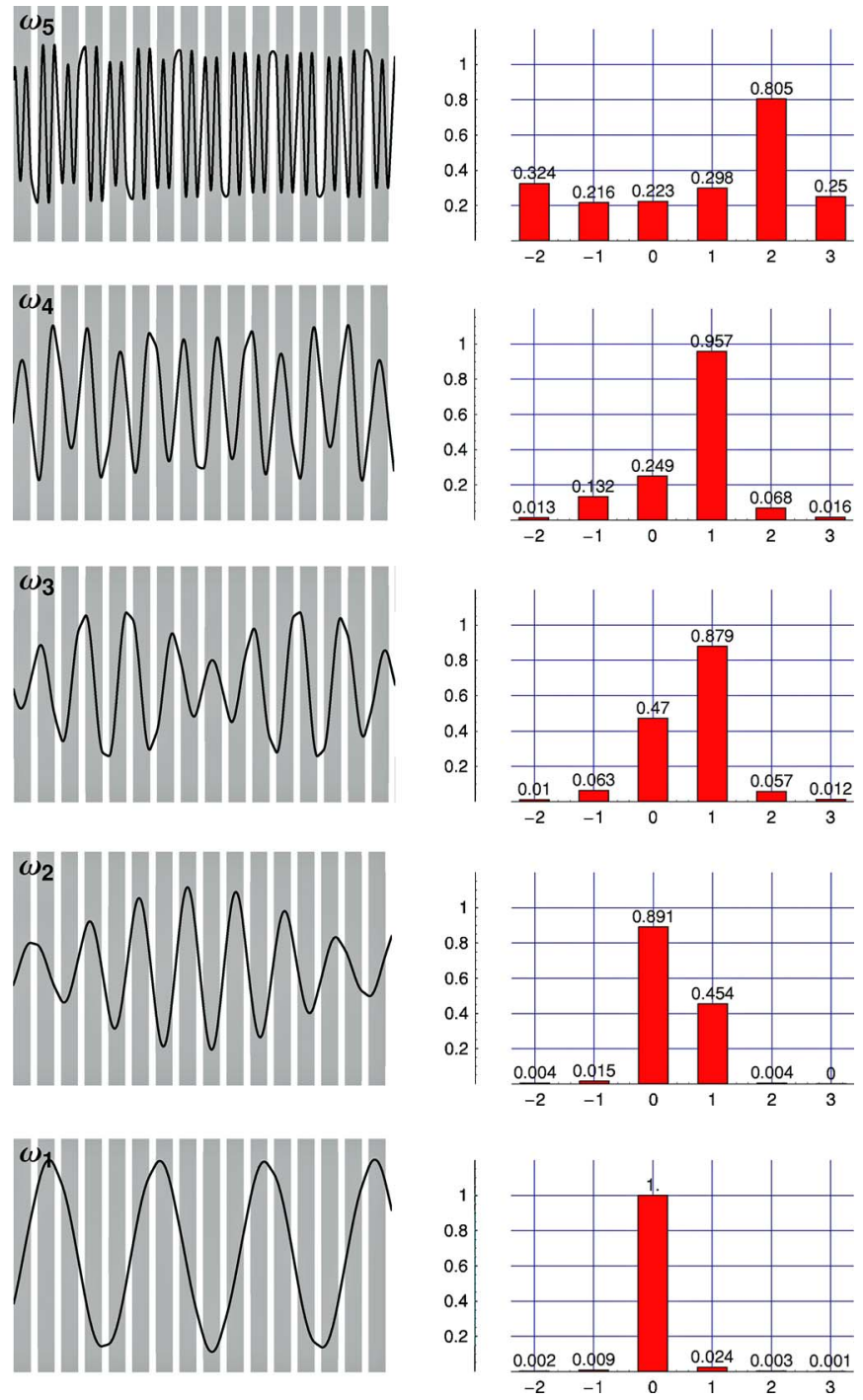

FIG. 3. (Color online) (Left) Field strength and (right) Fourier amplitudes of degenerate TE/TM Bloch waves propagating along the axis of symmetry of the illustrative structure with band diagram shown in Fig. 2. The wave parameters can be read off Fig. 2. Close to the band edge $\left(\omega_{2}, \omega_{3}\right)$ the counterpropagating components grow to make the wave increasingly similar to a standing wave with the field concentrated in the material of lower or higher refractive index depending on whether the frequency is close to the bottom or the top of a band gap. As the wavelength gets shorter, the waves deviate more and more from a plane wave even close to the centre of a band $\left(\omega_{5}\right)$.

$$
\frac{1}{n_{e}^{2}}=\frac{a}{\Lambda} \frac{1}{n_{a}^{2}}+\frac{b}{\lambda} \frac{1}{n_{b}^{2}}
$$

For phase matching between waves with different polarization in the long-wavelength limit, optimization of the birefringence can provide a method to choose the fill fractions of a structure.

Having gained some intuition on how the properties of Bloch waves depend on the photonic-crystal geometry and constituent materials we now turn to the problem of calculating the down-converted emission from real structures. 

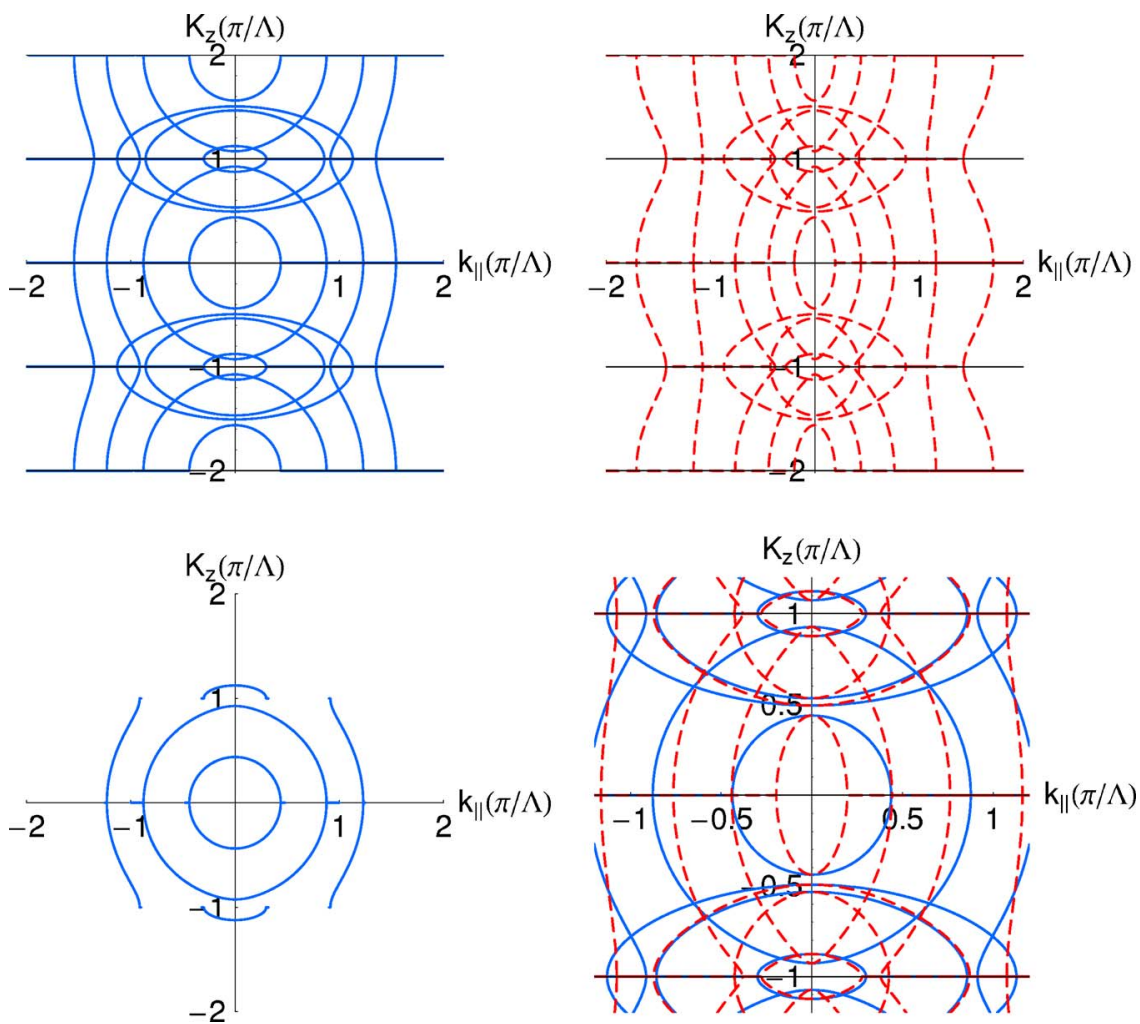

FIG. 4. (Color online) Dispersion surfaces for (top, left) TE and (top, right) TM Bloch waves with frequencies $\omega_{1, \ldots, 5}$. (Bottom, left) Dispersion surface for the leading TE wave components of waves $\omega_{1, \ldots, 3}$. (Bottom, right) The dispersion surfaces for TE and TM waves shown together. In the long wavelength limit the surfaces resemble those of a uniaxial birefringent crystal. As the wavelength is decreased to a length comparable to the periodicity of the structure, the surfaces increasingly distort.

\section{THE PHASE-MATCHING PROBLEM IN REAL STRUCTURES}

The parameter space for the design of a structure that achieves a particular type of phase matching is large. Here we give a procedure to make predictions for the emission. We suggest iterating the procedure, making changes to the structure guided by the intuition developed in the previous section, as a means of optimization. We take as an example a structure composed of 30 alternating layers of $\mathrm{Al}_{0.4} \mathrm{Ga}_{0.6} \mathrm{As}$ (123 nm thick) and air (64.5 $\mathrm{nm}$ thick) and consider down conversion of $750 \mathrm{~nm}$ photons to degenerate $1500 \mathrm{~nm}$ photon pairs. The fraction of aluminium was chosen to avoid absorption of the pump photons. The relative thickness of the layers was chosen to optimize the birefringence in the long wavelength limit. The periodicity was chosen to ensure both pump and down-converted photons would propagate, the pump in the second band, the down-converted photons in the first band. We proceed according to the following recipe.

(i) Band structure. We first plot the band diagram (Fig. 5) of the structure. An intuitive variable in terms of which to plot the band diagram is the angle $\theta$ of propagation of the plane-wave solution inside the material of lower refractive index which is given by $\sin (\theta)=k_{\|} / k_{z}$. When $\left|k_{\|} / k_{z}\right|>1$ total internal reflection occurs at the interfaces between the layers and the Bloch waves do not propagate along the crystal, but rather in a direction perpendicular to the crystal axis. In the case of the example structure this occurs when $k_{\|}$is equal to the magnitude of the free-space wave vector $\omega / c$. It is of course necessary to include natural absorption of the materials in the band diagram plot since it can restrict the accessible bands. $\mathrm{Al}_{0.4} \mathrm{Ga}_{0.6} \mathrm{As}$ absorbs at wavelengths below $640 \mathrm{~nm}$ [16]. The natural dispersion of the materials is ac- counted for by including the frequency dependence of $n_{a, b}$ (see Ref. [16] for AlGaAs) in Eq. (18).

(ii) Fourier spectrum. Having chosen frequencies that propagate (or having varied the periodicity to ensure they propagate), we look at the amplitude of the Fourier components of the Bloch waves having $k_{\|}=0$. Figure 6 shows the waves and Fourier components for our example structure. Both have a leading Fourier component. The phase-matched emission will in general not have $k_{\|}=0$. However if the combination $\omega k_{\|}=0$ does not lie close to a band edge, then the $k_{\|}=0$ spectrum is a good indication of what the $k_{\|} \neq 0$ spectrum will look like.

(iii) Phase matching of leading terms. We then look to satisfy the phase-matching equation $\mathbf{K}_{p}=\mathbf{K}_{1}+\mathbf{K}_{2}+\mathbf{G}$ starting with the leading $\mathbf{G}=n(2 \pi / \Lambda) \hat{\mathbf{z}}$ 's from the Fourier analysis above. This is done by drawing a dispersion diagram such as the one shown in Fig. 7. Intersections between the displaced down-converted photon dispersion surfaces represent Bloch vectors that phase-match according to Eq. (17).

The full set of solutions corresponds to the intersection of the full two-dimensional dispersion surfaces. However, the existence of an intersection in the diagrams discussed above is a necessary and sufficient condition for the two surfaces to have an intersection.

(iv) Plotting the emission. Having determined that the intersections exist, we look at the detailed direction of the emission. To do this analytically we make the simplifying assumption that the crystal transverse dimensions are infinite $\left(L_{x}, L_{y} \gg \lambda / n_{a, b}\right)$. This is both justified in most experimental situations and makes it possible to produce a manageable analytical prediction of the emission. The transverse part of the phase-matching function then becomes a product of two delta functions that ensure 


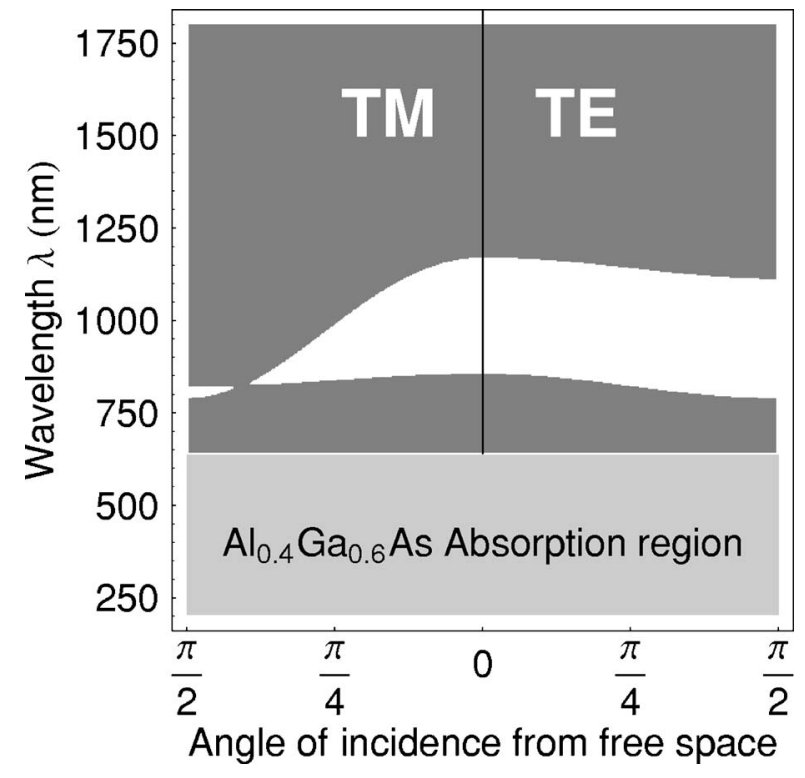

FIG. 5. Band diagram for the example $\mathrm{Al}_{0.4} \mathrm{Ga}_{0.6} \mathrm{As} /$ air structure with periodicity $\Lambda=187.5 \mathrm{~nm}$. Points in the grey bands correspond to propagating TM (left) and TE (right) states. Points in the white bands correspond to frequencies that cannot propagate in the structure. The shaded area below $640 \mathrm{~nm}$ represents the region in which $\mathrm{Al}_{0.4} \mathrm{Ga}_{0.6} \mathrm{As}$ is absorbing.

$$
\begin{gathered}
k_{x}^{(1)}=-k_{x}^{(2)}, \\
k_{y}^{(1)}=k_{y}^{(p)}-k_{y}^{(2)} .
\end{gathered}
$$

Substituting these relations into the longitudinal part of the phase-matching function [Eq. (16) with the $\delta$ function replaced by the appropriate sinc function] to eliminate either $k_{x, y}^{(1)}$ or $k_{x, y}^{(2)}$, we can then plot the phase-matching function for each emission. The elimination procedure is equivalent to calculating a partial trace over the emissions of the twin photon. It should be noted that the partial trace calculation is much simpler than in the case of birefringent nonlinear crystals such as BBO since the optic axis is by default aligned with the crystal axis. Figure 8 shows such a plot for three types of processes: Type I where a pump photon with TM polarization down converts to photons 1 and 2 with TE polarization: $\quad p(\mathrm{TM}) \rightarrow 1(\mathrm{TE})+2(\mathrm{TE}), \quad$ type $\quad \mathrm{II}: \quad p(\mathrm{TM})$ $\rightarrow 1(\mathrm{TE})+2(\mathrm{TM})$, and type III: $p(\mathrm{TM}) \rightarrow 1(\mathrm{TM})+2(\mathrm{TM})$. The width of the emission rings corresponds to the allowed longitudinal momentum mismatch and is inversely proportional to the crystal length. As can be seen in Fig. 8, it is an important parameter for the crystal size being considered here. For a discussion of the relation between the crystal thickness and the yield of entangled photon pairs in $\mathrm{BBO}$, see Ref. [17].

(v) Efficiency of the process. From the plots of the detailed emission, the efficiency for emission in directions of interest can be estimated. The values of $k_{\|}$for photons 1 and 2 are read off the plot. The amplitude for the phase-matched process can then be calculated by performing the sum over the Fourier coefficients [Eq. (16)], or by numerical integration of the field amplitudes in the nonlinear layers [Eq. (15)].
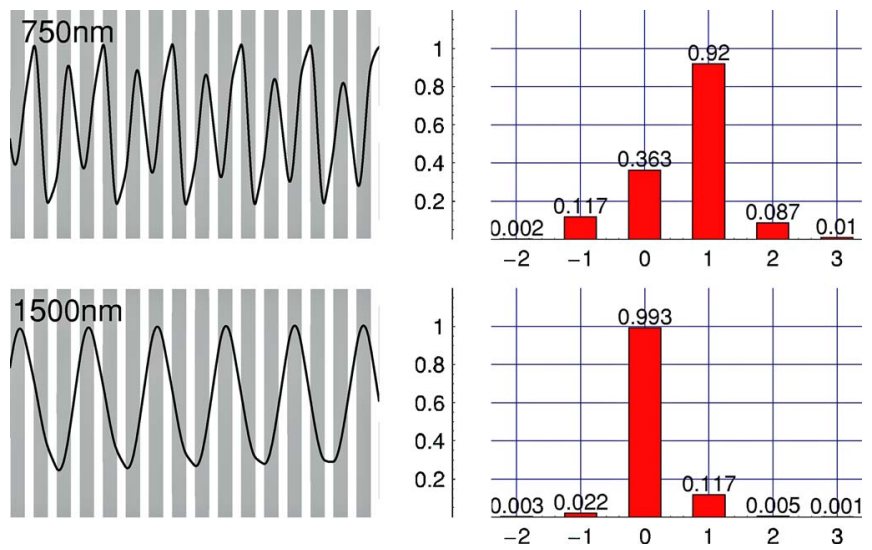

FIG. 6. (Color online) (Left) Field strength and (right) Fourier amplitudes of degenerate TE/TM Bloch waves propagating along the axis of symmetry of the example structure.

In the case of the Fourier amplitude calculation it will be necessary to use the coefficients $\tilde{\chi}(\mathbf{G})$ from the Fourier expansion of $\chi(r)$ which is

$$
\begin{aligned}
\chi^{(2)}(r)= & \frac{1}{\pi} \sum_{n} \frac{1}{n}\left[\chi_{b} e^{-i(b \pi n / \Lambda)} \sin \left(\frac{b \pi}{\Lambda} n\right)\right. \\
& \left.+\chi_{a} e^{-i(a \pi n / \Lambda)} \sin \left(\frac{a \pi}{\Lambda} n\right)\right] e^{i(2 \pi x / \Lambda) n}
\end{aligned}
$$

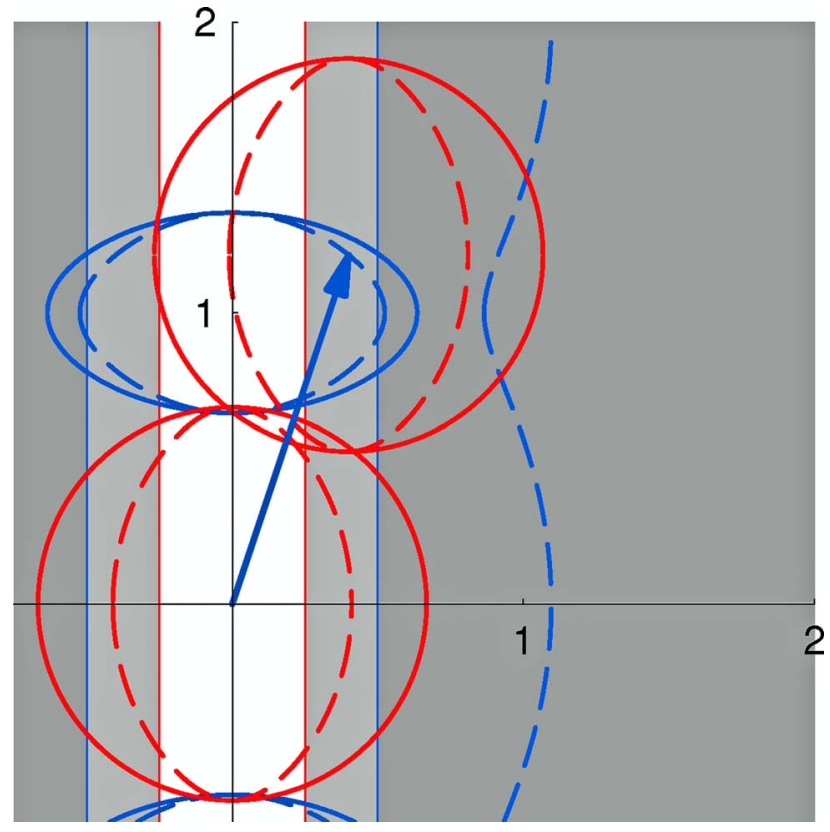

FIG. 7. (Color online) Diagram to determine whether downconverted light will phase match. The solid (dashed) blue lines represent the dispersion surfaces for pump $(\lambda=750 \mathrm{~nm}) \mathrm{TE}(\mathrm{TM})$ photons. The solid (dashed) red lines represent the dispersion surface for down-converted $(\lambda=1500 \mathrm{~nm}) \mathrm{TE}(\mathrm{TM})$ photons. The pump dispersion surface is centered at the origin, and the blue arrow represents the pump Bloch vector. Two down-converted photon dispersion surfaces are drawn, centered on the extremities of the blue arrow. Intersections between the down-converted photon surfaces represent Bloch vectors that phase-match according to Eq. (19). 

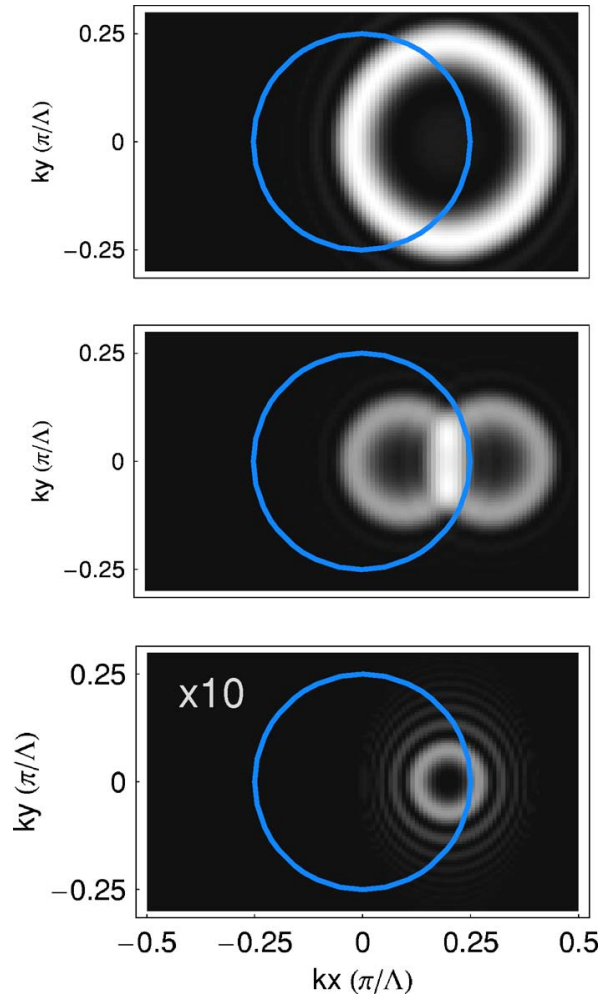

FIG. 8. (Color online) Three types of phase matching in the $\mathrm{Al}_{0.4} \mathrm{Ga}_{0.6} \mathrm{As} /$ air structure. (Top) type-I $(\mathrm{TM} \rightarrow \mathrm{TE}+\mathrm{TE})$, (middle) type II $(\mathrm{TM} \rightarrow \mathrm{TE}+\mathrm{TM})$, (bottom) type III $(\mathrm{TM} \rightarrow \mathrm{TM}+\mathrm{TM})$. The density plots in $k_{\|}$space represent the emission strength calculated using only the phase-matching function and do not include contributions from the tensor nature of the $\chi^{(2)}$ nonlinearity. The blue circle represents the values of $k_{\|}$that totally internally reflect between the layers. The type III phase matching is weak when compared to types I and II for this set of parameters and so was multiplied by a factor 10 to make it visible.

The $n=0$ term is simply the weighted average of the $\chi$ 's: $\left(a \chi_{a}+b \chi_{b}\right) / \Lambda$.

For photons emitted in the directions corresponding to the intersection of the rings in Fig. 8 (middle) or Fig. 9 there is one dominant combination of $\mathbf{G}^{\prime}$ s: $\mathbf{G}_{\chi}=0, \mathbf{G}_{p}=(2 \pi / \Lambda) \hat{\mathbf{z}}$, and $\mathbf{G}_{1}=\mathbf{G}_{2}=0$. For this combination the Fourier amplitudes are $0.66,0.90,0.99$, and 0.98 , respectively. The combined effect of the Fourier amplitudes leads to a factor 0.58.

In addition, the tensor nature of the $\chi^{(2)}$ interaction needs to be included. This is done by taking the contraction of the unit polarization vectors of the relevant Fourier components of $E_{p}, E_{1}, E_{2}$ with the $\chi^{(2)}$ tensor: $\chi_{i j k}^{(2)}\left[\hat{\varepsilon}_{p}^{b}\left(k_{x}^{p}, k_{y}^{p}\right)\right]^{i}\left[\hat{\varepsilon}_{(1)}^{b}\left(k_{x}^{(1)}, k_{y}^{(1)}\right)\right]^{j}\left[\hat{\varepsilon}_{(2)}^{b}\left(k_{x}^{(2)}, k_{y}^{(2)}\right)\right]^{k}$. The $\chi^{(2)}$ tensor of AlGaAs has $\overline{4} 3 m$ point group symmetry and has only three nonzero coefficients all having magnitude $200 \mathrm{pm} / \mathrm{V}$ [5]. For the conventional (100) surface orientation, the crystalline axis coincides with the direction of normal incidence that we have defined to be the $z$ direction. For photons emitted in the directions corresponding to the intersection of the rings in Fig. 8 (Middle) or Fig. 9 this leads to a value of $0.53 \times 200 \mathrm{pm} / \mathrm{V}$.

We are thus in a position to compare the efficiency of the process to that in BBO. The overall down-conversion effi-

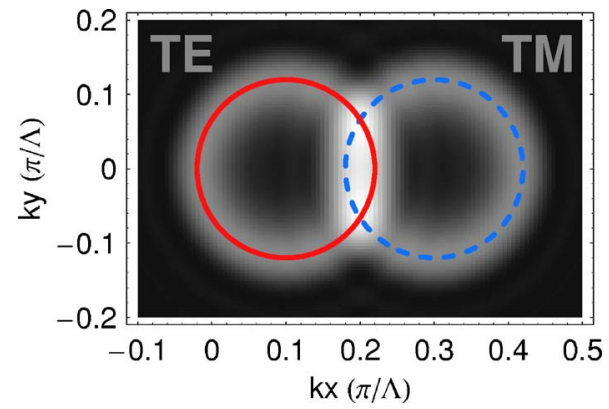

FIG. 9. (Color online) Polarization-entangled photon pairs can be extracted from the intersection of the TE and TM rings of the type II emission according to a well-known scheme $[3,18,19]$.

ciency for our example structure and choice of pump parameters is $(0.53 \times 0.58 \times 200)^{2} \sim 780$ times that in a BBO crystal of similar size, where we have assumed that there is no reduction of the $2.2 \mathrm{pm} / \mathrm{V}$ value of the $\mathrm{BBO} \chi^{(2)}$ coming from the tensor nature of the interaction.

It should be noted that there will also be down-conversion into directions that phase match but do not involve leading Fourier amplitudes. These can be treated in exactly the same way, but will have much lower amplitude.

(vi) Extraction of polarization-entangled photon pairs. To extract entangled photon pairs, we proceed as in the scheme for the extraction of entangled photons from nonlinear crystals [3], by collecting photon pairs from the intersection between the TE emission and the TM emission (see Fig. 9). At each intersection the photons will be polarized $\mathrm{TE}(\mathrm{H})$ or $\mathrm{TM}(\mathrm{V})$ with equal amplitude. However since the photons are emitted in TE/TM pairs, which ever the polarization of a photon at one intersection, we know the twin photon at the other intersection will have opposite polarization. Adding the amplitudes we obtain a maximally entangled polarization state:

$$
\frac{1}{\sqrt{2}}\left(|H\rangle|V\rangle+e^{i \Upsilon}|V\rangle|H\rangle\right),
$$

where $Y$ is a phase that can be easily tuned experimentally, for example, by placing a birefringent element in one of the paths.

The existence of a fixed phase relation between the two terms depends on the genuine impossibility of deducing the polarization of a photon collected at one of the intersections from any of its other properties. Such a coupling between degrees of freedom reduces the coherence between the terms. There are many techniques to recover the coherence, for example the use of compensating crystals [3] or of a polarizing beam splitter $[18,19]$.

\section{CONCLUSIONS}

We have presented a fully quantum-mechanical treatment of the down-conversion process in nonlinear photonic crystals, showing how to calculate the emission analytically in one dimensional structures. We have applied the theory to a realistic one-dimensional structure that consists of alternat- 
ing layers of $\mathrm{Al}_{0.4} \mathrm{Ga}_{0.6} \mathrm{As}$ and air and demonstrated that entangled photons with a wavelength of $1500 \mathrm{~nm}$ can be extracted from the down-conversion emission that results from the decay of pump photons having a wavelength of $750 \mathrm{~nm}$.

We now suggest some possible extensions of the present work. The results for the $\chi^{(2)}$ mediated emission of Sec. VI are valid for structures of one, two, and three dimensions. An obvious extension is thus the explicit analysis of Bloch-wave phase matching in two and three-dimensional structures. An advantage of the use of two-dimensional structures is that two-dimensional structures with a large index contrast can be more easily fabricated than one-dimensional structures with a large index contrast. Another interesting extension is the analysis of down conversion in the case where the pump photons arrive in short pulses or wave packets (see Ref. [20] for a discussion of the problem in $\mathrm{BBO}$ ). This brings up the topic of group velocity dispersion in photonic crystals which can differ significantly from its counterpart in natural crystals. It seems likely that the possibility of tuning this form of dispersion will provide interesting possibilities. Another interesting avenue of research might be to investigate the potential advantages of down converting to frequencies near a photonic band edge, where the density of states is considerably larger than that at the center of a band, thus further improving the efficiency. Finally it might be interesting to consider down conversion of frequencies close to the nonlinear material absorption band gap, where the nonlinearity can be significantly enhanced. In conclusion, the high degree of control afforded by the freedom of choosing the crystal geometry combined with the high nonlinearity of semiconductor materials for which many advanced fabrication techniques have been developed make nonlinear photonic crystals a promising source of entangled photon pairs.

\section{ACKNOWLEDGMENTS}

We acknowledge J.F. Hodelin for useful discussions and M. Rakher and F. Azhar for useful comments on the manuscript. This work was supported by NSF Grant No. PHY0304678 and DARPA Grant No. MDA972-01-1-0027.

\section{APPENDIX: DERIVATION OF THE BLOCH-WAVE AMPLITUDES}

In this appendix we derive an expression for the Fourier coefficients of the periodic part of the electric field in onedimensional Bragg structures. Throughout we will drop the $\mathrm{TE}(\mathrm{M})$ labels since the derivation is identical for the two cases. We begin by combining Eqs. (22) and (23) for the eigenamplitudes of the forward and backward moving components of the electric field in each layer, with the expression for the electric field in the $n$th layer [Eqs. (19) and (20)] to obtain

$$
\begin{aligned}
\mathbf{E}_{n}^{a}= & e^{i(\omega t-\mathbf{K} \cdot \mathbf{r})}\left[\left(a_{0}^{+} \hat{\varepsilon}_{+}^{a}\right) e^{-i k_{z}^{a}(z-n \Lambda)} e^{i \mathrm{~K}_{z}(z-n \Lambda)}\right. \\
& \left.+\left(a_{0}^{-} \hat{\varepsilon}_{-}^{a}\right) e^{i k_{z}^{a}(z-n \Lambda)} e^{i \mathrm{~K}_{z}(z-n \Lambda)}\right]
\end{aligned}
$$

$$
\begin{aligned}
\mathbf{E}_{n}^{b}= & e^{i(\omega t-\mathbf{K} \cdot \mathbf{r})}\left[\left(b_{0}^{+} \hat{\varepsilon}_{+}^{b}\right) e^{-i k_{z}^{b}(z-n \Lambda)} e^{i \mathrm{~K}_{z}(z-n \Lambda)}\right. \\
& \left.+\left(b_{0}^{-} \hat{\varepsilon}_{-}^{b}\right) e^{i k_{z}^{b}(z-n \Lambda)} e^{i \mathrm{~K}_{z}(z-n \Lambda)}\right] .
\end{aligned}
$$

Comparing these relations with Eq. (4) then yields the following relation:

$$
\begin{aligned}
\sum_{\mathbf{n}} \widetilde{\varepsilon}_{\mathbf{K}, \lambda}\left(n \frac{2 \pi}{\Lambda}\right) e^{i n(2 \pi / \Lambda) z}= & \sum_{\text {layers }} E_{+, n}^{a}(z) \hat{\varepsilon}_{+}^{a}+E_{-, n}^{a}(z) \hat{\varepsilon}_{-}^{a} \\
& +E_{+, n}^{b}(z) \hat{\varepsilon}_{+}^{b}+E_{-, n}^{b}(z) \hat{\varepsilon}_{-}^{b},
\end{aligned}
$$

where $E_{+/-}^{a(b)}(z)$ represent the electric field propagating in the forward/backward direction in material $a(b)$. In the $n$th layer they are given by

$$
\begin{aligned}
& E_{ \pm, n}^{a}(z)=a_{0}^{ \pm} e^{\mp i k_{z}^{a}(z-n \Lambda)} e^{i K_{z}(z-n \Lambda)}, \\
& E_{ \pm, n}^{b}(z)=b_{0}^{ \pm} e^{\mp i k_{z}^{b}(z-n \Lambda)} e^{i K_{z}(z-n \Lambda)} .
\end{aligned}
$$

To find the amplitudes of the of the Fourier coefficients $\widetilde{\varepsilon}_{\mathbf{K}, \lambda}$ we evaluate the Fourier transform of both sides of Eq. (A3). We define the Fourier transform $\tilde{f}$ of a function $f$ as

$$
\tilde{f}(q)=\frac{1}{\sqrt{2 \pi}} \int_{-\infty}^{\infty} d z f(z) e^{-i q z} .
$$

The Fourier transform of the left hand side of Eq. (A3) is.

$$
\sqrt{2 \pi} \sum_{\mathbf{n}} \widetilde{\varepsilon}_{\mathbf{K}, \lambda}\left(n \frac{2 \pi}{\Lambda}\right) \delta\left(q_{z}-n \frac{2 \pi}{\Lambda}\right) .
$$

To evaluate the Fourier transform of the right-hand side of Eq. (A3) it is convenient to first reexpress the sum over layers in terms of the following: the top hat function $T_{x_{1}, x_{2}}(x)$ :

$$
T_{x_{1}, x_{2}}(x)=\left\{\begin{array}{lc}
1, & x_{1} \leqslant x \leqslant x_{2}, \\
0, & x<x_{1}, x>x_{2}
\end{array}\right.
$$

with $x_{2}>x_{1}$, the comb function $\Pi_{(\Lambda, 2 N+1)}(z)$ :

$$
\Pi_{(\Lambda, 2 N+1)}(z)=\sum_{n=-N}^{N} \delta(z-n \Lambda),
$$

and the convolution of functions $f$ and $g, f * g$ :

$$
[f * g](x)=\int_{-\infty}^{\infty} d z f(z) g(x-z) .
$$

In terms of these functions we have

$$
\begin{gathered}
\sum_{\text {layers }} E_{+, n}^{a}(z) \hat{\varepsilon}_{+}^{a}+E_{-, n}^{a}(z) \hat{\varepsilon}_{-}^{a}+E_{+, n}^{b}(z) \hat{\varepsilon}_{+}^{b}+E_{-, n}^{b}(z) \hat{\varepsilon}_{-}^{b} \\
=E_{+}^{a}(z) \hat{\varepsilon}_{+}^{a}+E_{-}^{a}(z) \hat{\varepsilon}_{-}^{a}+E_{+}^{b}(z) \hat{\varepsilon}_{+}^{b}+E_{-}^{b}(z) \hat{\varepsilon}_{-}^{b}
\end{gathered}
$$

with

$$
E_{ \pm}^{a}(z)=\left[\left(E_{ \pm, n=0}^{a} T_{-a, 0}\right) * \prod_{(\Lambda, 2 N+1)}\right](z),
$$




$$
E_{ \pm}^{b}(z)=\left[\left(E_{ \pm, n=0}^{b} T_{-\Lambda,-a}\right) * \Pi_{(\Lambda, 2 N+1)}\right](z) .
$$

$E_{ \pm}^{a}(z)$ and $E_{ \pm}^{b}(z)$ are well defined functions at all points in the the crystal. They are equal to 0 outside layers $a$ and $b$ respectively and equal to $E_{ \pm, n}^{a}(z)\left[\mathrm{Eq}\right.$. (A4)] and $E_{ \pm, n}^{b}(z)$ [Eq. (A5)] in the $n$th layer. The Fourier transform of $E_{ \pm}^{a}(z)$ and $E_{ \pm}^{b}(z)$ can be simplified using the standard relation

$$
\widetilde{f * g}=\widetilde{f} \widetilde{g}
$$

to obtain

$$
\begin{aligned}
& \tilde{E}_{ \pm}^{a}=\left(E_{ \pm, n=0}^{a} T_{-a, 0}\right) \widetilde{\Pi}_{(\Lambda, 2 N+1)}, \\
& \widetilde{E}_{ \pm}^{b}=\left(E_{ \pm, n=0}^{b} \widetilde{T_{-\Lambda,-a}}\right) \widetilde{\Pi}_{(\Lambda, 2 N+1)} .
\end{aligned}
$$

If we evaluate the Fourier transform of $\Pi_{(\Lambda, 2 N+1)}$ :

$$
\left[\widetilde{\Pi}_{(\Lambda, 2 N+1)}\right]\left(q_{z}\right)=\frac{1}{\sqrt{2 \pi}} \frac{\left.\sin \left[q_{z} \frac{\Lambda}{2}(2 N+1)\right]\right)}{\sin \left[q_{z} \frac{\Lambda}{2}\right]},
$$

and take the limit of $N \rightarrow \infty$, we obtain

$$
\lim _{N \rightarrow \infty} \widetilde{\Pi}_{(\Lambda, 2 N+1)}=\sqrt{2 \pi} \Pi_{(2 \pi / \Lambda, \infty)} .
$$

Comparison in this limit with the left-hand side of Eq. (A6) then leads to the following expression for $\widetilde{\varepsilon}_{\mathbf{K}, \lambda}$ :

$$
\begin{aligned}
\widetilde{\varepsilon}_{\mathbf{K}, \lambda}\left(n \frac{2 \pi}{\Lambda}\right)= & E_{+, 0}^{a+T_{-a, 0}}\left(n \frac{2 \pi}{\Lambda}\right) \hat{\varepsilon}_{+}^{a}+E_{-, 0}^{a T_{-a, 0}}\left(n \frac{2 \pi}{\Lambda}\right) \hat{\varepsilon}_{-}^{a} \\
& +E_{+, 0}^{b} \widetilde{T_{-\Lambda,-a}}\left(n \frac{2 \pi}{\Lambda}\right) \hat{\varepsilon}_{+}^{b}+E_{-, 0}^{b} \widetilde{T_{-\Lambda,-a}}\left(n \frac{2 \pi}{\Lambda}\right) \hat{\varepsilon}_{-}^{b} .
\end{aligned}
$$

Evaluating $E_{ \pm, 0}^{a} \widetilde{T}_{-a, 0}\left(q_{z}\right)$ and $E_{ \pm, 0}^{b} \widetilde{T}_{-\Lambda,-a}\left(q_{z}\right)$ :

$$
E_{ \pm, 0}^{a} \widetilde{T}_{-a, 0}\left(q_{z}\right)=\frac{a}{\sqrt{2 \pi}} a_{0}^{ \pm} \frac{\sin \left[\left(K_{z} \mp k_{z}^{a}-q_{z}\right) \frac{a}{2}\right]}{\left(K_{z} \mp k_{z}^{a}-q_{z}\right) \frac{a}{2}} e^{-i\left(K_{z} \mp k_{z}^{a}-q_{z}\right)(a / 2)},
$$

$$
\begin{aligned}
E_{ \pm, 0}^{b} \widetilde{T_{-\Lambda,-a}}\left(q_{z}\right)= & \frac{b}{\sqrt{2 \pi}} b_{0}^{ \pm} \frac{\sin \left[\left(K_{z} \mp k_{z}^{b}-q_{z}\right) \frac{b}{2}\right]}{\left(K_{z} \mp k_{z}^{b}-q_{z}\right) \frac{b}{2}} \\
& \times e^{-i\left(K_{z} \mp k_{z}^{b}-q_{z}\right)(a+b / 2)}
\end{aligned}
$$

completes our derivation. Combining Eqs. (A15) and (A16) with Eq. (A14), we obtain the result given in Sec. V.
[1] D. Bouwmeester, A. Ekert, and A. Zeilinger, The Physics of Quantum Information (Springer, Berlin, 2000), and references therein.

[2] E. Knill, R. Laflamme, and G. J. Milburn, Nature (London) 409, 46 (2001).

[3] P. G. Kwiat, K. Mattle, H. Weinfurter, A. Zeilinger, A. V. Sergienko, and Y. Shih, Phys. Rev. Lett. 75, 4337 (1995).

[4] M. J. A. de Dood, W. T. M. Irvine, and D. Bouwmeester, Phys. Rev. Lett. 93, 040504 (2004).

[5] S. Bergfeld and W. Daum, Phys. Rev. Lett. 90, 036801 (2003).

[6] R. C. Eckardt, H. Masuda, Y. X. Fan, and R. L. Byer, IEEE J. Quantum Electron. 26, 922 (1990).

[7] J. P. van der Ziel, M. Ilegems, and R. M. Mikulyak, Appl. Phys. Lett. 28, 735 (1976).

[8] A. De Rossi, V. Berger, M. Calligaro, G. Leo, V. Ortiz, and X. Marcadet, Appl. Phys. Lett. 79, 3758 (2001).

[9] A. Fiore, V. Berger, E. Rosencher, P. Bravetti, and J. Nagle, Nature (London) 391, 463 (1998); J. P. Mondia, H. M. van Driel, W. Jiang, A. R. Cowan, and J. F. Young, Opt. Lett. 28, 2500 (2003).
[10] K. Sakoda and K. Ohtaka, Phys. Rev. B 54, 5742 (1996); J. N. Winn, S. Fan, J. D. Joannopoulos, and E. P. Ippen, ibid. 59, 1551 (1999); S. Saltiel and Y. S. Kivshar, Opt. Lett. 25, 1204 (2000).

[11] A. N. Vamivakas, B. E. A. Saleh, A. V. Sergienko, and M. C. Teich, Phys. Rev. A 70, 043810 (2004).

[12] A. Caticha and N. Caticha, Phys. Rev. B 46, 479 (1992).

[13] A. Yariv and P. Yeh, Optical Waves in Crystals (Wiley, New York, 2003).

[14] D. A. Kleinman, Phys. Rev. 174, 1027 (1968); C. K. Hong and L. Mandel, Phys. Rev. A 31, 2409 (1985).

[15] V. Berger, Phys. Rev. Lett. 81, 4136 (1998).

[16] D. E. Aspnes, S. M. Kelso, R. A. Logan, and R. Bhat, J. Appl. Phys. 60, 754 (1986).

[17] P. S. K. Lee, M. P. van Exter, and J. P. Woerdman, Phys. Rev. A 70, 043818 (2004).

[18] P. G. Kwiat, P. H. Eberhard, A. M. Steinberg, and R. Y. Chiao, Phys. Rev. A 49, 3209 (1994).

[19] Y.-H. Kim, S. P. Kulik, M. V. Chekhova, W. P. Grice, and Y. Shih, Phys. Rev. A 67, 010301(R) (2003).

[20] W. P. Grice and I. A. Walmsley, Phys. Rev. A 56, 1627 (1997). 